\title{
Atrial Fibrillation Analysis using Bessel Kernel based Time Frequency Distribution Technique
}

\author{
Sandun Kodituwakku, Thushara D. Abhayapala, Rodney A. Kennedy \\ Research School of Information Sciences and Engineering \\ The Australian National University, Australia
}

\begin{abstract}
We propose a Bessel kernel based time frequency distribution technique for identification and tracking of spectrum of Atrial Fibrillation (AF) in ECG. The algorithm shows a good frequency resolution and a low RMS error even when the noise dominates the signal which is critical for detecting the low amplitude AF activity within the ECG. In comparison with other time frequency distributions, the Bessel kernel reduces cross terms between frequencies in the multi-component ECG signal. Superiority of the Bessel kernel method over the short time Fourier transform (STFT) is demonstrated using a frequency modulated sinusoidal model and using real AF data. At low signal to noise levels the Bessel distribution outperforms the STFT and at an SNR of -5dB the RMS error is reduced from $1.8 \mathrm{~Hz}$ to $0.8 \mathrm{~Hz}$. Also it achieves a frequency resolution of $0.5 \mathrm{~Hz}$ at an SNR of OdB which is four times better than that of the STFT.
\end{abstract}

Keywords - Atrial Fibrillation, Bessel distribution, Time frequency analysis

\section{Introduction}

$\mathrm{AF}$ is the most common sustained cardiac arrhythmia, increasing in prevalence with age, accounting for approximately one third of hospitalizations for cardiac rhythm disturbances [1]. AF is characterized by the replacement of consistent $\mathrm{P}$ waves on the ECG by rapid oscillations (fibrillatory waves) that vary in amplitude, frequency, and shape, associated with an irregular ventricular response. AF affects approximately $10 \%$ of the population over age of 75 and is associated with an increased risk of stroke [1,2].

Previous studies have shown that spectrum of the atrial activity of the ECG under AF has a dominant peak (AF frequency) and there is a significant correlation between spontaneous or drug induced termination of $\mathrm{AF}$ and the time variation of AF frequency [3], indicating the importance of accurately tracking the AF frequency in time. Tracking of spectral content of a time signal can be done using STFT, but it has an inherent tradeoff between time and frequency resolution [4]. Stridh et al. [5,6] employed Wigner-Ville and Choi-Williams time frequency distributions for analyzing time variation of spectral content of AF, but the accuracy and noise robustness of the algorithms are inconclusive. As AF activity has a substantially low amplitude in the ECG and almost undistinguishable from noise and other ECG artifacts, it is important that a given method has a significant robustness to noise. Sandberg et al. [7] employed Hidden Markov models for frequency tracking of AF, but the performance at SNR less than OdB has not been studied. The proposed Bessel kernel based time frequency distribution technique is capable of tracking AF frequency in ECG with a good frequency resolution and a low RMS error even when the noise dominates the signal.

\section{System model}

Atrial fibrillation was mathematically modeled by a sum of frequency modulated sinusoidals with time varying amplitude and its harmonics $[7,8]$ and is given by

$$
s(t)=\sum_{k=1}^{M+1} a_{k}(t) \cos \left[k \omega_{0} t+\frac{\Delta \omega}{\omega_{f}} \sin \left(\omega_{f} t\right)\right]+n(t)
$$

where $a_{k}(t)=e^{-\gamma(k-1)}\left(a+\Delta a \sin \left(\omega_{a} t\right)\right), \omega_{0}$ is the fundamental AF frequency, $\omega_{f}$ is the frequency of frequency modulation, $\Delta \omega$ is the maximum frequency deviation, $M$ is the number of harmonics excluding the fundamental, $\gamma$ is the decaying factor of harmonics, $a$ is the average amplitude of the fundamental, $\omega_{a}$ is the frequency of amplitude modulation, and $\Delta a$ is the maximum amplitude deviation. $n(t)$ represents white Gaussian noise, artifacts from insufficient QRST cancelation, and other ECG artifacts. According to the model, AF frequency is given by

$$
\omega_{A F}(t)=\omega_{0}+\Delta \omega \cos \left(\omega_{f} t\right) .
$$

The objective is to accurately estimate $\omega_{A F}(t)$, especially when $n(t)$ is higher compared to the amplitude of the fundamental $a_{1}(t)$. Though this model is an approximation to the real AF signal, it is useful when analyzing the performance of different algorithms. A time window of the simulated AF signal is shown in Figure 1. 


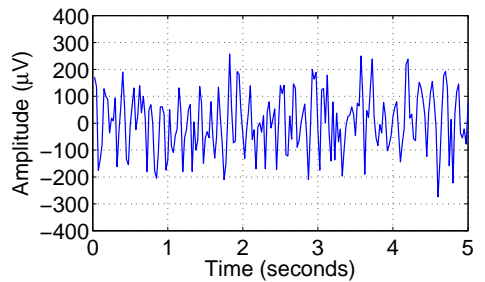

Figure 1. Time window of 5 second interval of simulated $\mathrm{AF}$ signal (1) at $\mathrm{SNR}=0 \mathrm{~dB}\left(\omega_{0}=2 \pi 7, \omega_{f}=2 \pi 0.1\right.$, $\Delta \omega=2 \pi 1, M=3, \gamma=1, a=100, \omega_{a}=2 \pi 0.08$, $\Delta a=10)$.

\section{Bessel distribution}

Cohen's class of time frequency distributions [4] is a well known method for joint time frequency analysis and is given by

$$
\begin{aligned}
C_{s}(t, \omega ; \phi) & =\frac{1}{2 \pi} \iiint e^{j \xi \mu-j \tau \omega-j \xi t} \phi(\xi, \tau) \\
& \times s\left(\mu+\frac{\tau}{2}\right) s^{*}\left(\mu-\frac{\tau}{2}\right) d \xi d \mu d \tau
\end{aligned}
$$

where $s(\mu)$ is the time signal to be analyzed, $t$ and $\omega$ are time and frequency respectively, and $\tau$ and $\xi$ are time lag and frequency lag respectively. Limits of each integral is from $-\infty$ to $\infty$. The kernel function $\phi(\xi, \tau)$ defines a particular distribution of the class.

The kernel of the Bessel distribution [9] is given by,

$$
\phi(\xi, \tau)=\frac{J_{1}(2 \pi \alpha \xi \tau)}{\pi \alpha \xi \tau}
$$

where $J_{1}(\cdot)$ is the Bessel function of first kind of order one and $\alpha>0$ is a scaling factor. The time and frequency support properties of the joint distribution are preserved under the condition $\alpha \leq 0.5$. The Bessel kernel for $\alpha=0.1$ is shown in Figure 2. Due to the nonlinear nature of Cohen's

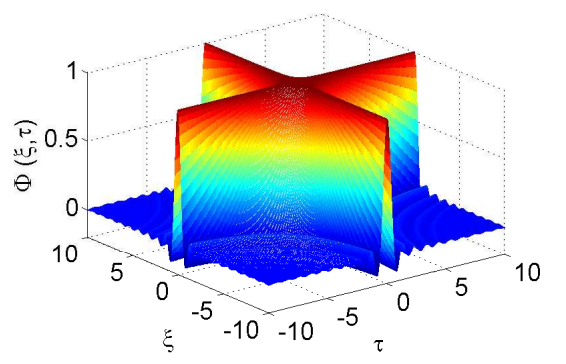

Figure 2. Bessel Kernel (4) for $\alpha=0.1$

class of time frequency distributions, cross terms appear in the distribution for a multi-component signal. The Bessel distribution is capable of smoothing out these cross terms, thus achieving a good frequency resolution-we illustrate this fact, first using a sum of two complex exponentials with constant amplitudes and then extend this idea to a sum of frequency modulated signals.

Let the time signal be given by $s(t)=A_{1} e^{j \omega_{1} t}+$ $A_{2} e^{j \omega_{2} t}$. Thus,

$$
\begin{array}{r}
s\left(\mu+\frac{\tau}{2}\right) s^{*}\left(\mu-\frac{\tau}{2}\right)=A_{1}^{2} e^{j \omega_{1} \tau}+A_{2}^{2} e^{j \omega_{2} \tau} \\
+2 A_{1} A_{2} e^{j\left(\omega_{1}+\omega_{2}\right) \frac{\tau}{2}} \cos \left(\omega_{1}-\omega_{2}\right) \mu .
\end{array}
$$

Using (3) we write the Bessel distribution as

$$
B_{s}(t, \omega)=B_{s, A T}^{(1)}(t, \omega)+B_{s, A T}^{(2)}(t, \omega)+B_{s, C T}(t, \omega)
$$

where $B_{s}(t, \omega)$ denotes the Bessel distribution, and $B_{s, A T}^{(\cdot)}(t, \omega)$ and $B_{s, C T}(t, \omega)$ are auto terms and cross term of the distribution respectively. The aim is to minimize the cross term without compromising the resolution of auto terms. From (3), (4) and (5), $B_{s, A T}^{(1)}(t, \omega)$ can be written as

$$
\begin{aligned}
B_{s, A T}^{(1)}(t, \omega) & =\frac{A_{1}^{2}}{2 \pi} \int e^{-j\left(\omega-\omega_{1}\right) \tau} \iint e^{j(\mu-t) \xi} \\
& \times \frac{J_{1}(2 \pi \alpha \xi \tau)}{\pi \alpha \xi \tau} d \xi d \mu d \tau \\
& =2 \pi A_{1}^{2} \delta\left(\omega-\omega_{1}\right)
\end{aligned}
$$

where $\delta(\cdot)$ is the Dirac delta function and similarly for $B_{s, A T}^{(2)}(t, \omega)$. The cross term $B_{s, C T}(t, \omega)$ is given by

$$
\begin{aligned}
& B_{s, C T}(t, \omega)=\frac{A_{1} A_{2}}{\pi} \int e^{-j\left(\omega-\frac{\omega_{1}+\omega_{2}}{2}\right) \tau} \iint e^{j(\mu-t) \xi} \\
& \times \frac{J_{1}(2 \pi \alpha \xi \tau)}{\pi \alpha \xi \tau} \cos \left(\omega_{1}-\omega_{2}\right) \mu d \xi d \mu d \tau \\
& =\frac{8 A_{1} A_{2}}{\alpha \omega_{d}} \cos \omega_{d} t \sqrt{1-\frac{\left(\omega-\omega_{m}\right)^{2}}{\alpha^{2} \omega_{d}^{2}} \Pi\left(\frac{\omega-\omega_{m}}{2 \alpha \omega_{d}}\right)}
\end{aligned}
$$

where $\omega_{m}=\left(\omega_{1}+\omega_{2}\right) / 2, \omega_{d}=\left|\omega_{1}-\omega_{2}\right|$ and $\Pi(\cdot)$ is the rectangle function. At a given time $t=t_{0},(10)$ is a half an ellipse centered at $\left(\omega_{m}, 0\right)$ and has semi axes $\left(8 A_{1} A_{2}\right) /\left(\alpha \omega_{d}\right) \cos \omega_{d} t_{0}$ and $\alpha \omega_{d}$. Thus energy distribution of the cross term can be controlled by varying $\alpha$, as shown in Figure 3. Cross term energy distribution derived in (10) is a good approximation for a sum of frequency modulated signals as shown in Figure 4 provided that, $\left\|\max \left(\Delta \omega_{1}, \Delta \omega_{2}\right)\right\| \ll\left\|\omega_{1}-\omega_{2}\right\|$ where

$$
\begin{aligned}
s(t) & =A_{1} \cos \left[\omega_{1} t+\frac{\Delta \omega_{1}}{\omega_{f 1}} \sin \left(\omega_{f 1} t\right)\right] \\
& +A_{2} \cos \left[\omega_{2} t+\frac{\Delta \omega_{2}}{\omega_{f 2}} \sin \left(\omega_{f 2} t\right)\right] .
\end{aligned}
$$

With the above evidence, we assert that the method is well suited for analyzing time variation of AF frequency under the influence of noise, as the AF model comprises of sum of frequency modulated signals. 

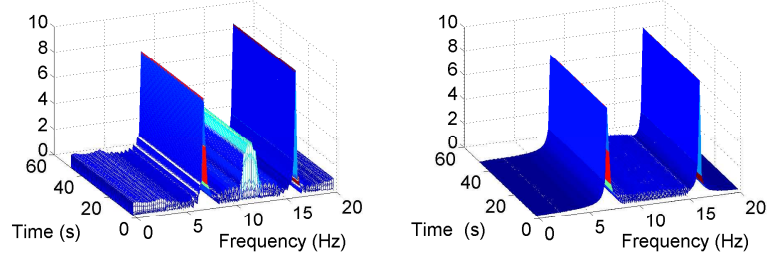

Figure 3. Bessel Distribution for sum of two complex exponentials (a) $\alpha=0.1$, (b) $\alpha=0.4$
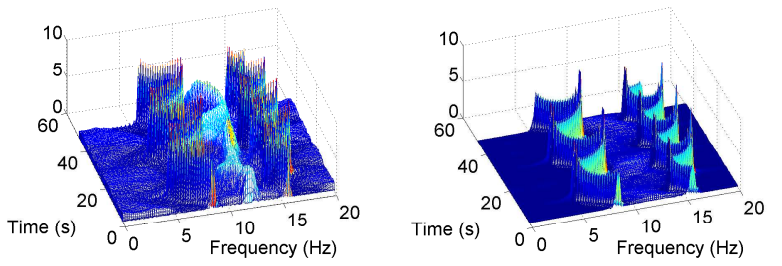

Figure 4. Bessel Distribution for sum of two frequency modulated signals (a) $\alpha=0.1$, (b) $\alpha=0.4$

\section{Results}

Simulations were performed using (1) to generate AF signals where additive white Gaussian noise (AWGN) was used for $n(t)$ at different SNR settings. Numerical implementation of the Bessel distribution can be found in [9]. $\alpha$ was set to 0.5 in order to minimize the cross term effects and at the same time preserving time and frequency support properties of the distribution. In order to analyze the performance of the Bessel distribution it was compared to the STFT. Results for SNR $=5 \mathrm{~dB}, 0 \mathrm{~dB},-5 \mathrm{~dB}$ are shown in Figures 5, 6, and 7 respectively. The results were analyzed using two performance measuring criteria, RMS error and frequency resolution. RMS Error was used as the first per-
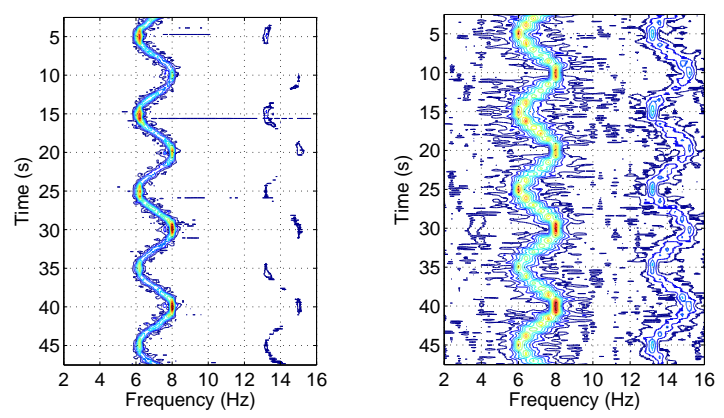

Figure 5. Bessel distribution vs STFT for simulated AF under $\mathrm{AWGN}$ at $\mathrm{SNR}=5 \mathrm{~dB}$.

formance measuring criteria of the algorithm. It measures the noise robustness and accuracy of a particular method in estimating the $\mathrm{AF}$ frequency under a noisy environment
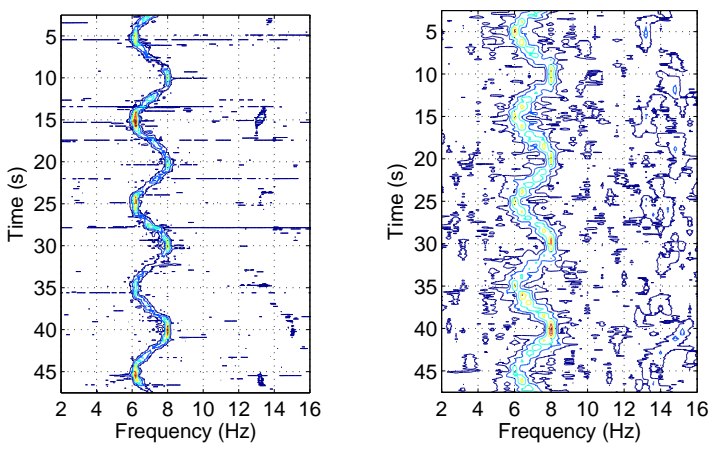

Figure 6. Bessel distribution vs STFT for simulated AF under $\mathrm{AWGN}$ at $\mathrm{SNR}=0 \mathrm{~dB}$.
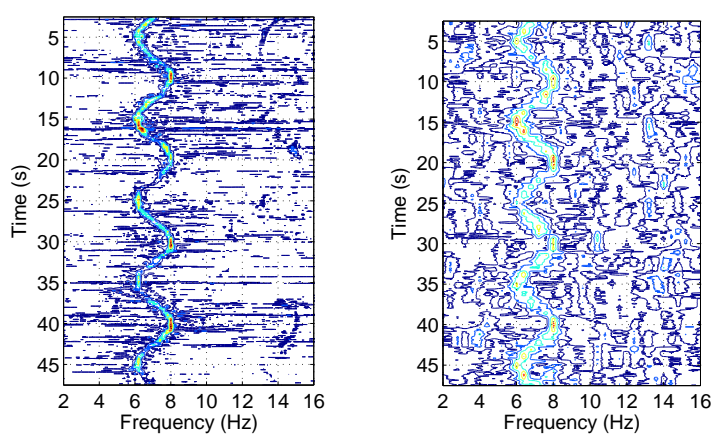

Figure 7. Bessel distribution vs STFT for simulated AF under $\mathrm{AWGN}$ at $\mathrm{SNR}=-5 \mathrm{~dB}$.

and is given by

$$
E_{R M S}=\sqrt{\left\langle\left[\omega_{N}(t)-\omega_{A F}(t)\right]^{2}\right\rangle}
$$

where $\omega_{N}(t)$ is the estimated AF frequency using time frequency distribution under noise, $\omega_{A F}(t)$ is the $\mathrm{AF}$ frequency and $\langle\cdot\rangle$ is the average over time. Simulation results for RMS Error is given in Figure 8.

Frequency resolution was used as the second performance measuring criteria and is estimated by time average of $3 \mathrm{~dB}$ Bandwidth of the distribution. It is another measure of noise robustness in terms of the energy concentration under a noisy environment and is given by

$$
\int_{\omega_{\max }(t)-\frac{1}{2} \omega_{r}(t)}^{\omega_{\max }(t)+\frac{1}{2} \omega_{r}(t)} P_{s}(t, \omega) d \omega=0.5 \int_{-\infty}^{\infty} P_{s}(t, \omega) d \omega
$$

where $\omega_{r}(t)$ is the frequency resolution, $\omega_{\max }(t)$ is the frequency with maximum energy and $P_{s}(t, \omega)$ is a particular time frequency distribution. Frequency resolution at a particular SNR setting will be given by $\left\langle\omega_{r}(t)\right\rangle$. Simulation results for frequency resolution is given in Figure 9.

At low signal to noise levels the Bessel distribution outperformed the STFT and at an SNR of -5dB the RMS error 


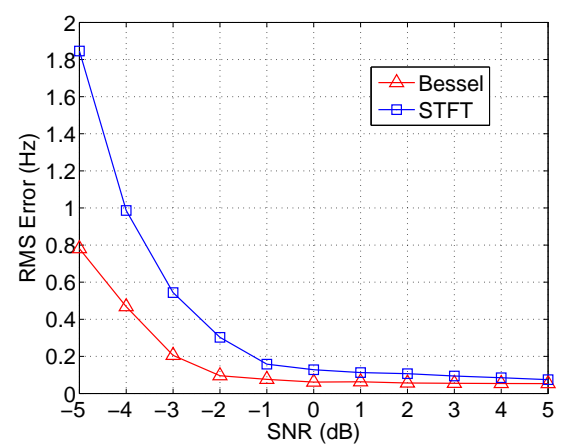

Figure 8. RMS Error of Bessel distribution and STFT for simulated AF with AWGN.

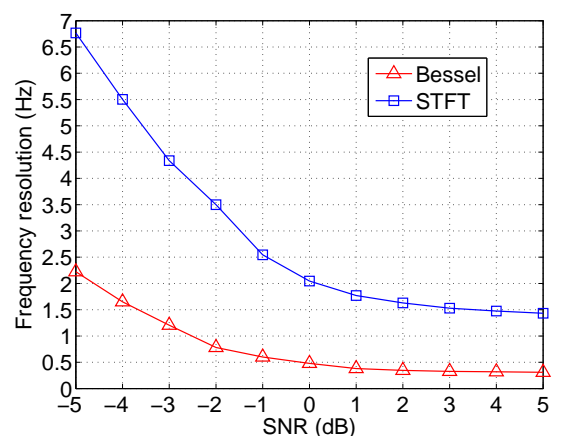

Figure 9. Frequency resolution of Bessel distribution and STFT for simulated AF with AWGN.

was reduced from $1.8 \mathrm{~Hz}$ to $0.8 \mathrm{~Hz}$. Also it achieved a frequency resolution of $0.5 \mathrm{~Hz}$ at an SNR of $0 \mathrm{~dB}$ which is four times better than that of the STFT.

In Figure 10, we present an example of real AF signal taken from PhysioBank [10] AF Termination Challenge Database in order to show the applicability and superiority of the method.

\section{Future work}

Future work includes parameterizing Bessel distribution for paroxysmal and persistent $\mathrm{AF}$ conditions, identifying patterns in the distribution under pharmacological therapy and direct current cardioversion, and generalizing the Bessel distribution for other supraventricular tachyarrhythmias including Atrial Flutter.

\section{References}

[1] Fuster V, Ryden LE, et al. Acc/aha/esc 2006 guidelines for the management of patients with atrial fibrillation. Amer $\mathrm{J}$ Cardiol Aug 2006;48(4):854-906.

[2] Singh SN, Tang XC, et al. Quality of life and exercise performance in patients in sinus rhythm versus persistent atrial fibrillation. Amer J Cardiol Aug 2006;48(4):721-730.
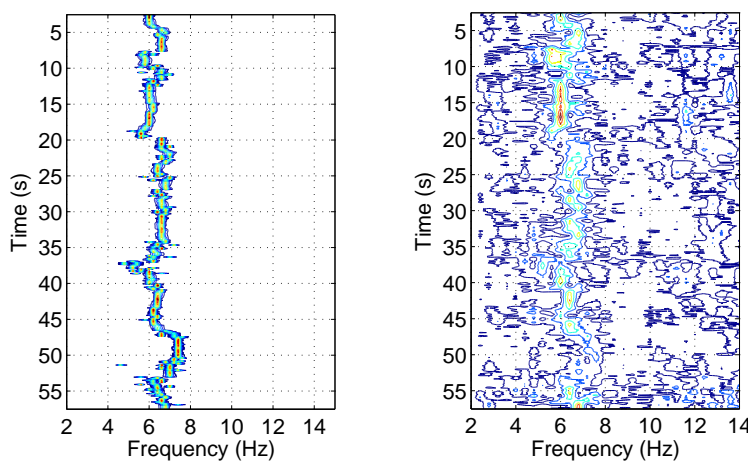

Figure 10. Bessel distribution vs STFT for ECG Record n02 of PhysioBank AF Termination Challenge Database.

[3] Bollmann A, Kanuru NA, McTeague KK, Walter PF, DeLurgio DB, Langberg JJ. Frequency analysis of human atrial fibrillation using the surface electrocardiogram and its response to ibutilide. Amer J Cardiol Jan 1998;81:1439_ 1445.

[4] Cohen L. Time-frequency distributions-a review. IEEE Proc Jul 1989;77(7):941-981.

[5] Stridh M, Sornmo L, Olsson SB, Sattar F. Spectral analysis and time-dependent properties of atrial fibrillation in the surface ecg. Proc of IEEE Med and Bio 1998;20(1):112114.

[6] Stridh M, Sornmo L, Meurling CJ, Olsson SB. Characterization of atrial fibrillation using the surface ecg: Timedependent spectral properties. IEEE Trans Biomed Eng Jan 2001;48(1):19-27.

[7] Sandberg F, Stridh M. Frequency tracking of atrial fibrillation using hidden markov models. IEEE Trans Biomed Eng Feb 2008;55(2):502-511.

[8] Stridh M, Sornmo L. Spatiotemporal QRST cancellation techniques for analysis of atrial fibrillation. IEEE Trans Biomed Eng Jan 2001;48(1):105-111.

[9] Guo Z, Durand L, Lee HC. The time-frequency distributions of nonstationary signals based on a bessel kernel. IEEE Trans Signal Proc Jul 1994;42(7):1700-1707.

[10] Goldberger AL, Amaral LAN, Glass L, Hausdorff JM, Ivanov PC, Mark RG, Mietus JE, Moody GB, Peng CK, Stanley HE. PhysioBank, PhysioToolkit, and PhysioNet: Components of a new research resource for complex physiologic signals. Circulation 2000 (June 13);101(23):e215-e220. Circulation Electronic Pages: http://circ.ahajournals.org/cgi/content/full/101/23/e215.

Address for correspondence:

Sandun Kodituwakku

RSISE, ANU, ACT 0200, Australia

Email: sandun.kodituwakku@anu.edu.au 